\title{
Some results in topology optimization applied to biomechanics
}

\author{
G. Machado ${ }^{a, *}$, L. Trabucho ${ }^{b}$ \\ a Department of Mathematics for Science and Technology, University of Minho, 4800-058 Guimarães, Portugal \\ ${ }^{\mathrm{b}}$ Department of Mathematics, University of Lisbon, 1649-003 Lisbon, Portugal
}

Received 10 March 2004; accepted 12 March 2004

Available online 8 June 2004

\begin{abstract}
This paper presents the application of a topology optimization algorithm based in homogenization theory. Three examples in structural design will be solved numerically. The first two are formulated such that analytical solutions can also be developed. To obtain this goal, the microscopic structure that we considered is formed of laminates because for this type of composite materials there is an explicit dependence of the homogenized coefficients on the design variables. The last example regards bone remodelling. Here, where it is impossible to obtain the analytical solution, the applied algorithm produces numerical results which are in good agreement with Wolff's Law.
\end{abstract}

(c) 2004 Elsevier Ltd. All rights reserved.

Keywords: Biomechanics; Bone remodelling; Elasticity; Homogenization theory; Optimality conditions

\section{Introduction}

In 1892 the German physiologist Julius Wolff proposed an explanation for the distribution of cortical bone and cancellous bone, actually known as Wolff's Law [15]. Basically it states that bone has the ability to remodel, by changing its size, shape, and structure, to meet the mechanical demands placed on it. It also says that bone remodelling is a kind of an optimization problem, in the sense that bone distributes in a way that maximizes its stiffness, while the trabeculae will orient along major stress lines [13].

The algorithm introduced by Bendsøe and Kikuchi in 1988 [5] for topology optimization problems when applied to the determination of bone distribution seems to recover Wolff's conjectures. There, the first introduced novelty was the transformation of the initial problem into a material distribution problem, where composite materials were used as the base material. Another novelty was the application of Homogenization Theory (e.g. $[6,10])$ to determine the macroscopic material properties

\footnotetext{
* Corresponding author.

E-mail address: gjm@mct.uminho.pt (G. Machado).
}

from the microscopic material constituents (cf. $[1,3,7,11])$. This approach to solve remodelling problems is followed e.g. in $[2,9,12]$.

In order to better understand the algorithm, we wish to apply it to simple examples where it is possible to develop analytical solutions, starting with the unidimensional case - bending of a bar-in Section 2. Section 3 considers elasticity problems. The first example of this section, with simple geometry and loads, is still capable of developing analytical solutions to compare with the obtained numerical results. The second one, is the application of the algorithm to bone remodelling.

\section{Bending of a bar}

\subsection{Problem formulation}

Consider a rod occupying the interval $\Omega=(0, L) \subset$ $\mathbb{R}$, with cross section area $A$ and with second moment of area with respect to a principal axes perpendicular to the bending plane of the rod denoted by $I$. Consider that the rod is subjected to a transverse load of intensity $f$ over its domain $\Omega$, clamped at $x=0$ and also subjected to a transversal tip force $F$ at $x=L$. 
Let us assume that the material the rod is made of possesses a microstructure formed by the layered combination of two homogeneous and isotropic materials: one, strong and expensive, with Young's modulus $E^{+}$, specific mass $\rho^{+}$and proportion $\tau$, with $0 \leqslant \tau \leqslant 1$; the other, weak and cheap, with Young's modulus $E^{-}\left(<E^{+}\right)$, specific mass $\rho^{-}\left(<\rho^{+}\right)$and proportion $1-\tau$; we assume that $f$ is independent of the specific mass, i.e., $\partial f / \partial \rho=0$.

We will consider a laminated microstructure oriented along a system of axes $O y_{1} y_{2}$ such that $O y_{1}$ is parallel to $O x_{1}$ and $O y_{2}$ is parallel to $O x_{2}$ (left-hand side of Fig. 1). If we apply the Homogenization Theory [4], the homogenized or macroscopic Young's modulus has the following expression:

$E_{1}^{\mathrm{H}}=\frac{E^{+} E^{-}}{\tau E^{-}+(1-\tau) E^{+}}$.

If we consider, instead, that the layer's orientation is defined by axes $O y_{1}$ parallel to $O x_{2}$ and $O y_{2}$ parallel to $O x_{1}$ (right-hand side of Fig. 1), the expression of the homogenized Young's modulus is

$E_{2}^{\mathrm{H}}=\tau E^{+}+(1-\tau) E^{-}$.

In both situations, the expression for the homogenized specific mass is given by

$\rho^{\mathrm{H}}=\tau \rho^{+}+(1-\tau) \rho^{-}$.

The equilibrium equation the displacement field $u$ has to satisfy, in its weak form, is given by

$a\left(\tau ; u^{\tau}, v\right)=\ell(v) \quad \forall v \in V$,

where

$$
\begin{aligned}
& a(\tau ; u, v)=\int_{0}^{L} E^{\mathrm{H}}(\tau) I \frac{\mathrm{d}^{2} u}{\mathrm{~d} x^{2}} \frac{\mathrm{d}^{2} v}{\mathrm{~d} x^{2}} \mathrm{~d} x, \\
& \ell(v)=\int_{0}^{L} f v \mathrm{~d} x+F v(L),
\end{aligned}
$$
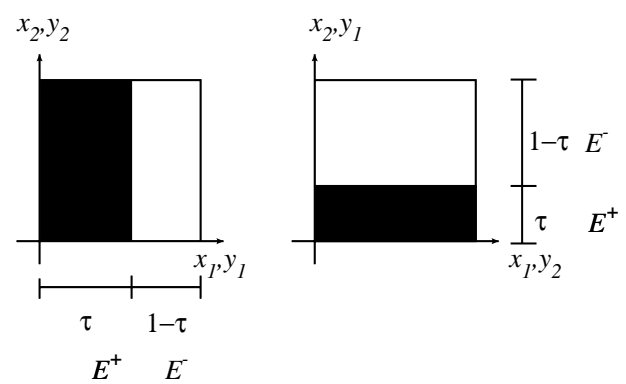

Fig. 1. Rank-1 layered microstructure, where the system of axes $O y_{1} y_{2}$ represents the microscopic level and $O x_{1} x_{2}$ the macroscopic level.
$V=\left\{v \in H^{2}(\Omega): v(0)=\frac{\mathrm{d} v}{\mathrm{~d} x}(0)=0\right\}$,

$V$ denotes the space of kinematically admissible displacement fields and $E^{\mathrm{H}}$ the homogenized Young's modulus independently of the laminated orientation.

The fact that for fixed control $\tau$ the problem possesses a unique solution $u^{\tau}$ relies on standard results. In fact for a given $\tau \in L^{\infty}(\Omega,[0,1])$, the functional $a(\tau ; \cdot, \cdot)$ is bilinear, continuous and coercive. On the other hand, functional $\ell(\cdot)$ is linear and continuous and the conclusion follows from Lax-Milgram's Lemma.

The problem we are addressing considers as objective function the work of the applied forces plus a term which penalizes the stiffest and more expensive material $E^{+}$. We want to determine the function $\tau$ which minimizes this objective function, subject to the equilibrium equation and to the lower and upper bounds in $\tau$. The problem formulation is the following:

$$
\begin{array}{ll}
\min _{\tau} & j(\tau) \\
\text { s.t.: } & a\left(\tau ; u^{\tau}, v\right)=\ell(v) \quad \forall v \in V, \\
& 0 \leqslant \tau \leqslant 1
\end{array}
$$

with

$j(\tau)=\int_{0}^{L} f u^{\tau} \mathrm{d} x+F u^{\tau}(L)+k \int_{0}^{L} \rho^{\mathrm{H}} A \mathrm{~d} x$,

where the positive constant $k$ represents the work done in order to add to the rod a unit of mass. We should note that this formulation is equivalent to the maximization of the stiffness of the solid, taking into account the cost penalization.

\subsection{Analytical solution}

In order to solve the problem under consideration, we construct the Lagrangian

$$
\begin{aligned}
\mathscr{L}= & j(\tau)+\lambda\left(a\left(\tau ; u^{\tau}, v\right)-\ell(v)\right) \\
& +\int_{0}^{L} \tau^{+}(\tau-1) \mathrm{d} x-\int_{0}^{L} \tau^{-} \tau \mathrm{d} x,
\end{aligned}
$$

where $\tau^{+}, \tau^{-}$and $\lambda$ are the Lagrange multipliers associated to constraints $\tau \leqslant 1, \tau \geqslant 0$ and the equilibrium equation, respectively. From the necessary conditions of stationarity one obtains the following conditions for a.e. $x \in \Omega$ and for all $v \in V$ :

$v=\frac{1}{\lambda} u^{\tau}$,

$a\left(\tau ; u^{\tau}, v\right)=\ell(v)$,

$\tau^{+} \geqslant 0, \quad \tau^{+}(\tau-1)=0$,

$\tau^{-} \geqslant 0, \quad \tau^{-} \tau=0$, 
$k \frac{\mathrm{d} \rho^{\mathrm{H}}}{\mathrm{d} \tau} A-\frac{\mathrm{d} E^{\mathrm{H}}}{\mathrm{d} \tau} I\left(\frac{\mathrm{d}^{2} u^{\tau}}{\mathrm{d} x^{2}}\right)^{2}+\tau^{+}-\tau^{-}=0$.

If Eq. (7) is rewritten as

$\chi_{\tau}+\tau^{+}-\tau^{-}=0$

$\chi_{\tau}=k \frac{\mathrm{d} \rho^{\mathrm{H}}}{\mathrm{d} \tau} A-\frac{\mathrm{d} E^{\mathrm{H}}}{\mathrm{d} \tau} I\left(\frac{\mathrm{d}^{2} u^{\tau}}{\mathrm{d} x^{2}}\right)^{2}$,

and considering (5) and (6), we have

$$
\begin{cases}\chi_{\tau}<0 \Rightarrow \tau^{+}>0, & \tau^{-}=0 \Rightarrow \tau=1, \\ \chi_{\tau}=0 \Rightarrow \tau^{+}=0, & \tau^{-}=0 \Rightarrow \tau \in[0,1], \\ \chi_{\tau}>0 \Rightarrow \tau^{+}=0, & \tau^{-}>0 \Rightarrow \tau=0 .\end{cases}
$$

So, as $k, \mathrm{~d} \rho^{\mathrm{H}} / \mathrm{d} \tau, A, \mathrm{~d} E^{\mathrm{H}} / \mathrm{d} \tau, I$ and $\left(\mathrm{d}^{2} u^{\tau} / \mathrm{d} x^{2}\right)^{2}$ are all positive terms, if the cost $k$ is sufficiently low we just have the stiffest material; if the cost is too high, we just have the weakest material; for intermediate values of $k$, we have a real laminate with a mixture of the two base materials.

Call, now, $\mathscr{F}(x)$ the total applied force at $x$, i.e.,

$\mathscr{F}(x)=\int_{x}^{L}\left(F+\int_{t}^{L} f \mathrm{~d} s\right) \mathrm{d} t$.

Considering the natural boundary conditions at $x=L$

$E^{\mathrm{H}} I \frac{\mathrm{d}^{2} u}{\mathrm{~d} x^{2}}=0 \quad$ and $\quad-\frac{\mathrm{d}}{\mathrm{d} x}\left(E^{\mathrm{H}} I \frac{\mathrm{d}^{2} u}{\mathrm{~d} x^{2}}\right)=F$,

and integrating twice the differential expression of the equilibrium equation

$\frac{\mathrm{d}^{2}}{\mathrm{~d} x^{2}}\left(E^{\mathrm{H}} I \frac{\mathrm{d}^{2} u}{\mathrm{~d} x^{2}}\right)=f$,

one obtains

$E^{\mathrm{H}} I \frac{\mathrm{d}^{2} u}{\mathrm{~d} x^{2}}=\mathscr{F}$.

Finally, this last expression and (1), (2) and (9), lead to

$\chi_{\tau, E_{1}^{\mathrm{H}}}=k\left(\rho^{+}-\rho^{-}\right) A-\frac{\left(E^{+}-E^{-}\right) \mathscr{F}^{2}}{E^{+} E^{-} I}$,

$\chi_{\tau, E_{2}^{\mathrm{H}}}=k\left(\rho^{+}-\rho^{-}\right) A-\frac{\left(E^{+}-E^{-}\right) \mathscr{F}^{2}}{\left(\tau E^{+}+(1-\tau) E^{-}\right)^{2} I}$.

These expressions will enable us to determine the analytical solutions of the examples presented in Section 2.4.

\subsection{Numerical solution}

In order to solve the problem numerically, we use the finite element method.

We start by discretizing the domain $\Omega$ in a finite element mesh, where we assume a constant value for the design variable $\tau$ in each finite element. As an iterative algorithm, an initial approximation for $\tau$ in each finite element should be given, after what we can compute the homogenized elastic properties. Then, we determine an approximation for the displacement field $u$. A new approximation of $\tau$ is calculated in each element and a stopping criteria is tested (we consider as stopping criteria the condition $\left\|\tau^{(n+1)}-\tau^{(n)}\right\|_{\infty} \leqslant \epsilon$, where $\tau^{(n)}$ and $\tau^{(n+1)}$ are the approximation vectors to $\tau$ at iterations $(n)$ and $(n+1)$, respectively). If the criteria is satisfied, the iterative process is stopped. Otherwise, the process restarts where a new approximation to $\tau$ is calculated.

From (10), the update scheme for the design variable $\tau$ should satisfy the following conditions:

$$
\left\{\begin{array}{l}
\chi_{\tau_{e, p-1}}<0 \Rightarrow \tau_{e, p} \geqslant \tau_{e, p-1}, \\
\chi_{\tau_{e, p-1}}=0 \Rightarrow \tau_{e, p}=\tau_{e, p-1}, \\
\chi_{\tau_{e, p-1}}>0 \Rightarrow \tau_{e, p} \leqslant \tau_{e, p-1}
\end{array}\right.
$$

where we denote by $\tau_{e, p}$ the value of $\tau$ in iteration $p$ and at element $e$. We note that for intermediate densities $\left(0<\tau<1, \tau^{+}=\tau^{-}=0\right)$, Eq. (9) can be written as

$\bar{\chi}_{\tau}=\frac{1}{k \frac{\mathrm{d} \rho^{\mathrm{H}}}{\mathrm{d} \tau} A} \frac{\mathrm{d} E^{\mathrm{H}}}{\mathrm{d} \tau} I\left(\frac{\mathrm{d} u^{\tau}}{\mathrm{d} x}\right)^{2}$.

Combining these two last considerations, [4] proposed the following fixed-point update algorithm for $\tau_{e, p}$ :

- if $\tau_{e, p-1}\left(\bar{\chi}_{p-1}\right)^{\eta} \leqslant \max \left\{(1-\zeta) \tau_{e, p-1}, 0\right\}: \tau_{e, p}=\max \{(1-$

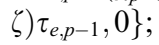

- if $\max \left\{(1-\zeta) \tau_{e, p-1}, 0\right\} \leqslant \tau_{e, p-1}\left(\bar{\chi}_{e, p-1}\right)^{\eta} \leqslant \min \{(1+$ ५) $\left.\tau_{e, p-1}, 1\right\}: \tau_{e, p}=\tau_{e, p-1}\left(\bar{\chi}_{p-1}\right)^{\eta}$

- if $\min \left\{(1+\zeta) \tau_{e, p-1}, 1\right\} \leqslant \tau_{e, p-1}\left(\bar{\chi}_{p-1}\right)^{\eta}: \tau_{e, p}=\min \{(1+$ ५) $\left.\tau_{e, p-1}, 1\right\}$,

where $\eta$ is a weighting factor and $\zeta$ is a move limit to control design changes between iterations (in our computations we consider $\eta=0.8$ and $\zeta=0.5$ ).

\subsection{Examples}

Let us consider a bar with constant square cross section of side $1\left(I=\frac{1}{12}\right)$, length $L=10$ and subjected to the loads $f=\sin \left(\frac{2 \pi x}{L}\right)$ and $F=\frac{L}{2 \pi}$. Since the major role is played by the ratio between homologous quantities, the properties of the base materials were chosen to be $E^{+}=2, \rho^{+}=2, E^{-}=1$ and $\rho^{-}=1$. However different values could have been considered.

In Table 1 we compare the analytical solutions developed in the previous section with the obtained numerical results for two values of $k$, as well as for the two orientations of the layers of the microstructure. For each value of $k$ and for each type of microstructure, the numerical solution is presented as the top bar and the analytical solution, $\bar{\tau}$, as the bottom bar. Elements where $\bar{\tau}$ is not constant are represented by theirs middle point. 
Table 1

Numerical and analytical solution for $k=100$ (first line) and $k=8$ (second line)

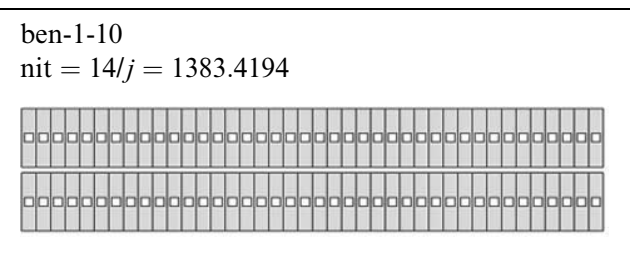

ben-2-10

nit $=29 / j=335.8419$

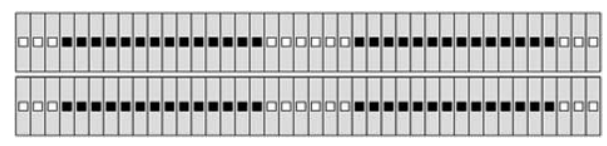

ben-1-11

nit $=29 / j=1383.4009$

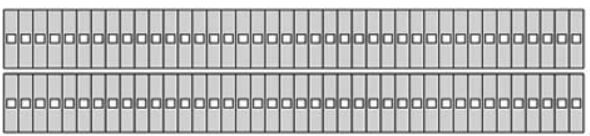

ben-2-11

nit $=24 / j=334.9749$

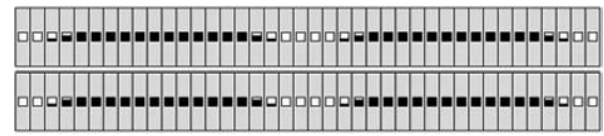

In all numerical tests we used $40 C^{1}$ cubic elements with $\tau^{(0)}=0.5$.

We identify each tested case by a sequence formed of three parts: the first part, "ben", identifies the problem type; the second one indicates the order in the sequence of the values of $k$; the last one identifies if the laminated microstructure is oriented along a system of axes $O y_{1} y_{2}$ such that $O y_{1}$ is parallel to $O x_{1}$ and $O y_{2}$ is parallel to $O x_{2}$ - suffix 10 - or if $O y_{1}$ is parallel to $O x_{2}$ and $O y_{2}$ is parallel to $O x_{1}$-suffix 11 .

For each example, we also indicate the number of iterations the process took to reach convergence (nit) and the value of the objective function $(j)$.

Considering $E_{1}^{\mathrm{H}}$, the analytical solution is given by:

- if $0 \leqslant k \leqslant \frac{3 L^{4}}{8 \pi^{4}}$ and $\left(\left(x^{*}<x<\frac{L}{2}-x^{*}\right)\right.$ or $\left(\frac{L}{2}+x^{*}<\right.$ $\left.\left.x<L-x^{*}\right)\right)$, with $x^{*}=\frac{L}{2 \pi} \arcsin \left(\sqrt{\frac{8 k}{3}} \frac{\pi^{2}}{L^{2}}\right): \bar{\tau}(x)=1$;

- otherwise: $\bar{\tau}(x)=0$.

Considering $E_{2}^{\mathrm{H}}$, we have:

- if $k=0: \bar{\tau}(x)=1$;

- otherwise: $\bar{\tau}(x)=\max \left(0, \min \left(1, \tau^{*}\right)\right)$, with $\tau^{*}=\sqrt{\frac{3}{4 k}} \frac{L^{2}}{\pi^{2}}$ $\left|\sin \left(\frac{2 \pi x}{L}\right)\right|-1$.

It is visible that there is a perfect agreement between the numerical results and the analytical solution.

\section{Linearized elasticity}

\subsection{Problem definition}

During this section, we will use the usual notation in plane elasticity theory, where greek indices take the values 1 and 2 . Although we will only consider the twodimensional case, it should be noted that everything that will follow has an immediate generalization to the threedimensional case. The summation convention on repeated indices will also be assumed.

Let us consider a solid occupying volume $\Omega$, an open bounded simply-connected subset of $\mathbb{R}^{2}$, with surface $\partial \Omega$. Moreover, consider that the body is fixed in a part of its surface, $\Gamma_{0}$, and that we have $\partial \Omega=\overline{\Gamma_{0}} \cup \overline{\Gamma_{1}}, \Gamma_{0} \cap$ $\Gamma_{1}=\emptyset$. Let $f=\left(f_{\alpha}\right)$ and $g=\left(g_{\alpha}\right)$, denote the force per unit volume and the force per unit surface area applied to the body, respectively.

Let us assume, again, that the material the solid is made of possesses a laminated microstructure formed by two base materials. The characteristics of these materials are the same as in the previous section, that is, homogeneous and isotropic of Young's modulus $E^{+}$and $E^{-}$ and with specific mass $\rho^{+}$and $\rho^{-}$, respectively, both with Poisson's ratio $v$, where we still have $E^{+}>E^{-}$and $\rho^{+}>\rho^{-}$.

We will consider two kinds of microstructure: in the first one, the material represented by the pair $\left(E^{+}, \rho^{+}\right)$is vertically intercalated with the material represented by the pair $\left(E^{-}, \rho^{-}\right)$, in the proportions $\tau$ and $1-\tau$, respectively, with $0 \leqslant \tau \leqslant 1$-this is called the rank-1 microstructure (left-hand side of Fig. 2); in the second one, the material represented by the pair $\left(E^{+}, \rho^{+}\right)$is
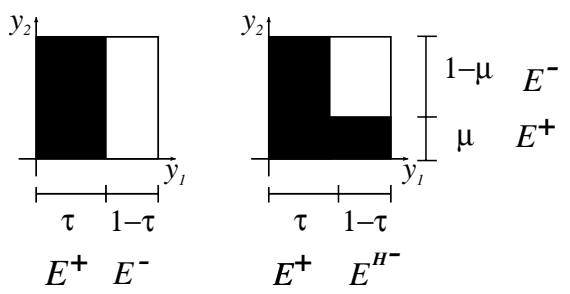

Fig. 2. Rank-1 and rank-2 layered microstructure, where the system of axes $O y_{1} y_{2}$ represents the microscopic level. 
again vertically intercalated with proportions $\tau$ and $1-\tau$, but this time with a new material, that we identify by the pair $\left(E^{H-}, \rho^{H-}\right)$; this new material is a rank-1, formed once again with the two base materials with proportions $\mu$ and $1-\mu(0 \leqslant \mu \leqslant 1)$, being the two scales of layers orthogonal-this is called the rank-2 microstructure (right-hand side of Fig. 2).

If we apply the Homogenization Theory [4], the nonnull homogenized elasticity coefficients have the following expressions:

$$
\begin{aligned}
\bar{E}_{1111}^{\mathrm{H}}= & \frac{E_{1111}^{+} E_{1111}^{-}}{\tau E_{1111}^{-}+(1-\tau) E_{1111}^{+}}, \\
\bar{E}_{1122}^{\mathrm{H}}= & \left(\tau \frac{E_{1122}^{+}}{E_{1111}^{+}}+(1-\tau) \frac{E_{1122}^{-}}{E_{1111}^{-}}\right) \bar{E}_{1111}^{\mathrm{H}}, \\
\bar{E}_{2222}^{\mathrm{H}}= & \tau E_{2222}^{+}+(1-\tau) E_{2222}^{-} \\
& -\left(\tau \frac{\left(E_{1122}^{+}\right)^{2}}{E_{1111}^{+}}+(1-\tau) \frac{\left(E_{1122}^{-}\right)^{2}}{E_{1111}^{-}}\right) \\
& +\left(\tau \frac{E_{1122}^{+}}{E_{1111}^{+}}+(1-\tau) \frac{E_{1122}^{-}}{E_{1111}^{-}}\right)^{2} \bar{E}_{1111}^{\mathrm{H}}, \\
\bar{E}_{1212}^{\mathrm{H}}= & \frac{E_{1212}^{+} E_{1212}^{-}}{\tau E_{1212}^{-}+(1-\tau) E_{1212}^{+}},
\end{aligned}
$$

where, in plane stress, we have for the non-null coefficients $E_{\alpha \beta \gamma \delta}^{+}$(both for rank-1 and rank-2 microstructures):

$$
\begin{array}{ll}
E_{1111}^{+}=\frac{E^{+}}{1-v^{2}}, & E_{1122}^{+}=\frac{v E^{+}}{1-v^{2}}, \\
E_{2222}^{+}=\frac{E^{+}}{1-v^{2}}, & E_{1212}^{+}=\frac{E^{+}}{2(1+v)},
\end{array}
$$

and for the non-null coefficients $E_{\alpha \beta \gamma \delta}^{-}$the expressions:

$$
\begin{array}{ll}
E_{1111}^{-}=\frac{E^{-}}{1-v^{2}}, & E_{1122}^{-}=\frac{v E^{-}}{1-v^{2}}, \\
E_{2222}^{-}=\frac{E^{-}}{1-v^{2}}, & E_{1212}^{-}=\frac{E^{-}}{2(1+v)},
\end{array}
$$

for a rank-1 microstructure and

$$
\begin{aligned}
& E_{1111}^{-}=I_{2}+\frac{v^{2} I_{1}}{1-v^{2}}, \quad E_{1122}^{-}=\frac{v I_{1}}{1-v^{2}}, \\
& E_{2222}^{-}=\frac{I_{1}}{1-v^{2}}, \quad E_{1212}^{-}=\frac{I_{1}}{2(1+v)},
\end{aligned}
$$

for a rank-2 microstructure, and where

$$
I_{1}=\frac{E^{+} E^{-}}{\mu E^{-}+(1-\mu) E^{+}}, \quad I_{2}=\mu E^{+}+(1-\mu) E^{-} .
$$

(If instead of plane stress, we have plane deformation, we just have to alter the expressions (12)-(14), but qualitatively there is no difference between these two situations.)
If we consider an angle $\theta$ made by the microstructure reference axes $O y_{1} y_{2}$ with respect to the macroscopic axes $O x_{1} x_{2}$, the homogenized elasticity coefficients are given by [4]

$E_{\alpha \beta \gamma \delta}^{\mathrm{H}}(\tau, \mu, \theta)=\bar{E}_{\varepsilon \zeta \eta \xi \xi}^{\mathrm{H}}(\tau, \mu) R_{\alpha \varepsilon} R_{\beta \zeta} R_{\gamma \eta} R_{\delta \xi}$,

where

$R=\left(\begin{array}{cc}\cos \theta & \sin \theta \\ -\sin \theta & \cos \theta\end{array}\right)$.

The homogenized specific mass is given by

$\rho^{\mathrm{H}}(\tau)=\tau \rho^{+}+(1-\tau) \rho^{-}$

if a rank-1 microstructure is considered, or by

$\rho^{\mathrm{H}}(\tau, \mu)=\rho^{+}(\tau+(1-\tau) \mu)+\rho^{-}(1-\tau)(1-\mu)$

if rank-2 microstructure is considered.

Denoting the independent variable by $x=\left(x_{\alpha}\right)$, the displacement field by $u=\left(u_{\alpha}\right)$, the strain tensor by $e=\left(e_{\alpha \beta}\right)$, where $e_{\alpha \beta}=\frac{1}{2}\left(\frac{\partial u_{\alpha}}{\partial x_{\beta}}+\frac{\partial u_{\beta}}{\partial x_{\alpha}}\right)$, and the stress tensor by $\sigma=\left(\sigma_{\alpha \beta}\right)$, where $\sigma_{\alpha \beta}=E_{\alpha \beta \gamma \delta} e_{\gamma \delta}$, the equilibrium equations that the displacement field $u$ has to satisfy, in its weak form, are given by

$a\left(\tau, \mu, \theta ; u^{\tau \mu \theta}, v\right)=\ell(v) \quad \forall v \in V$,

where

$a(\tau, \mu, \theta ; u, v)=\int_{\Omega} E_{\alpha \beta \gamma \delta}^{\mathrm{H}}(\tau, \mu, \theta) e_{\gamma \delta}(u) e_{\alpha \beta}(v) \mathrm{d} x$,

$\ell(v)=\int_{\Omega} f_{\alpha} v_{\alpha} \mathrm{d} x+\int_{\Gamma_{1}} g_{\alpha} v_{\alpha} \mathrm{d} s$,

$V=\left\{v \in\left[H^{1}(\Omega)\right]^{2}: v_{\alpha}=0\right.$ on $\left.\Gamma_{0}\right\}$.

The fact that for fixed controls $\tau, \mu$ and $\theta$ the elasticity problem possesses a unique solution $u^{\tau \mu \theta}$ relies on standard results in elasticity theory. In fact for a given $\tau, \mu \in L^{\infty}(\Omega,[0,1])$ and $\theta \in L^{\infty}(\Omega,[-\pi / 2, \pi / 2])$, the functional $a(\tau, \mu, \theta ; \cdot, \cdot)$ is bilinear, continuous and coercive, due to Korn's inequality. On the other hand, functional $\ell(\cdot)$ is linear and continuous and the conclusion follows from Lax-Milgram's Lemma.

The optimization problem, equivalent to the one presented in the previous section, can be stated as

$$
\begin{array}{ll}
\min _{\tau, \mu, \theta} & j(\tau, \mu, \theta) \\
\text { s.t. : } & a\left(\tau, \mu, \theta ; u^{\tau \mu \theta}, v\right)=\ell(v) \quad \forall v \in V, \\
& 0 \leqslant \tau \leqslant 1, \\
& 0 \leqslant \mu \leqslant 1,
\end{array}
$$


where

$$
\begin{aligned}
j(\tau, \mu, \theta)= & \int_{\Omega} f_{\alpha} u_{\alpha}^{\tau \mu \theta} \mathrm{d} x+\int_{\Gamma_{1}} g_{\alpha} u_{\alpha}^{\tau \mu \theta} \mathrm{d} s \\
& +k \int_{\Omega} \rho^{\mathrm{H}}(\tau, \mu) \mathrm{d} x .
\end{aligned}
$$

\subsection{Analytical solution}

In order to solve the problem under consideration, and for a rank-2 microstructure with rotation, we construct the Lagrangian

$$
\begin{aligned}
\mathscr{L}= & j(\tau, \mu, \theta)+\lambda\left(a\left(\tau, \mu, \theta ; u^{\tau \mu \theta}, v\right)-\ell(v)\right) \\
& +\int_{\Omega} \tau^{+}(\tau-1) \mathrm{d} x-\int_{\Omega} \tau^{-} \tau \mathrm{d} x \\
& +\int_{\Omega} \mu^{+}(\mu-1) \mathrm{d} x-\int_{\Omega} \mu^{-} \mu \mathrm{d} x
\end{aligned}
$$

where $\tau^{+}, \tau^{-}, \mu^{+}, \mu^{-}$and $\lambda$ are the Lagrange multipliers associated to constraints $\tau \leqslant 1, \tau \geqslant 0, \mu \leqslant 1, \mu \geqslant 0$ and the equilibrium equation, respectively. From the necessary conditions of stationarity one obtains the following conditions for a.e. $x \in \Omega$ and for all $v \in V$ :

$v=\frac{1}{\lambda} u^{\tau \mu \theta}$,

$a\left(\tau, \mu, \theta ; u^{\tau \mu \theta}, v\right)=\ell(v)$,

$\tau^{+} \geqslant 0, \quad \tau^{+}(\tau-1)=0$,

$\tau^{-} \geqslant 0, \quad \tau^{-} \tau=0$,

$\mu^{+} \geqslant 0, \quad \mu^{+}(\mu-1)=0$,

$\mu^{-} \geqslant 0, \quad \mu^{-} \mu=0$,

$k \frac{\partial \rho^{\mathrm{H}}}{\partial \tau}-\frac{\partial E_{\alpha \beta \gamma \delta}^{\mathrm{H}}}{\partial \tau} e_{\gamma \delta}\left(u^{\tau \mu \theta}\right) e_{\alpha \beta}\left(u^{\tau \mu \theta}\right)+\tau^{+}-\tau^{-}=0$,

$k \frac{\partial \rho^{\mathrm{H}}}{\partial \mu}-\frac{\partial E_{\alpha \beta \gamma \delta}^{\mathrm{H}}}{\partial \mu} e_{\gamma \delta}\left(u^{\tau \mu \theta}\right) e_{\alpha \beta}\left(u^{\tau \mu \theta}\right)+\mu^{+}-\mu^{-}=0$,

$\frac{\partial E_{\alpha \beta \gamma \delta}^{\mathrm{H}}}{\partial \theta} e_{\gamma \delta}\left(u^{\tau \mu \theta}\right) e_{\alpha \beta}\left(u^{\tau \mu \theta}\right)=0$.

The procedure to treat the design variables $\tau$ and $\mu$ is similar to the one presented in the previous section. Relatively to $\theta$ and following [4], if $\alpha$ denotes the angle formed by the principal strain axes with the macroscopic system of axes $O x_{1} x_{2}$ and $\psi$ the angle of rotation of the material frame $O y_{1} y_{2}$ with respect to the principal strain axes (we denote by $e_{\mathrm{I}}$ and $e_{\mathrm{II}}$ the principal strains), that is if $\theta=\alpha+\psi$, then, from (25), it can be shown that, if $e_{\mathrm{I}}=e_{\mathrm{II}}$, then $\psi$ can take any value (in this case, we consider $\theta=0$ ); if $e_{\mathrm{I}} \neq e_{\mathrm{II}}$, then $\sin (2 \psi)=0 \quad$ or $\quad \cos (2 \psi)=-\frac{\alpha}{\beta} \frac{e_{\mathrm{I}}+e_{\mathrm{II}}}{e_{\mathrm{I}}-e_{\mathrm{II}}}$,

where

$$
\begin{aligned}
& \alpha=\bar{E}_{1111}^{\mathrm{H}}-\bar{E}_{2222}^{\mathrm{H}}, \\
& \beta=\bar{E}_{1111}^{\mathrm{H}}+\bar{E}_{2222}^{\mathrm{H}}-2 \bar{E}_{1122}^{\mathrm{H}}-4 \bar{E}_{1212}^{\mathrm{H}},
\end{aligned}
$$

and one should choose the value that maximizes the strain energy

$\frac{1}{2} E_{\alpha \beta \gamma \delta}^{\mathrm{H}} e_{\alpha \beta} e_{\gamma \delta}$.

\subsection{Numerical solution}

The numerical discretization and update scheme is an adaptation of what is described in Section 2.3. We should also refer that in order to avoid checkerboard patterns, which are usual in this type of problems when four-node quadrilateral elements are involved, in the application of the finite element method we use isoparametric $C^{0}$ quadrilateral elements with nine nodes in Section 3.4.1 and with eight nodes in Section 3.4.2 (cf. [8]).

\subsection{Examples}

\subsubsection{Plane stress}

Consider a rectangular plate with principal axes $O x_{1}$ and $O x_{2}$ subjected to uniform loads on its boundary$\sigma_{11}=\bar{\sigma}_{11}, \sigma_{22}=\bar{\sigma}_{22}$ and $\sigma_{12}=\bar{\sigma}_{12}$-in a plane stress state (Fig. 3).

If we a consider a rank-1 layered microstructure without rotation, the strain field for a homogeneous solution obtained from the constitutive equations $\sigma_{\alpha \beta}=E_{\alpha \beta \gamma \delta} e_{\gamma \delta}$, is given by

$$
e_{11}\left(u^{\tau}\right)=\bar{\sigma}_{11}\left(\frac{1-v^{2}}{I_{1}}+\frac{v^{2}}{I_{2}}\right)-\frac{\bar{\sigma}_{22} v}{I_{2}}
$$

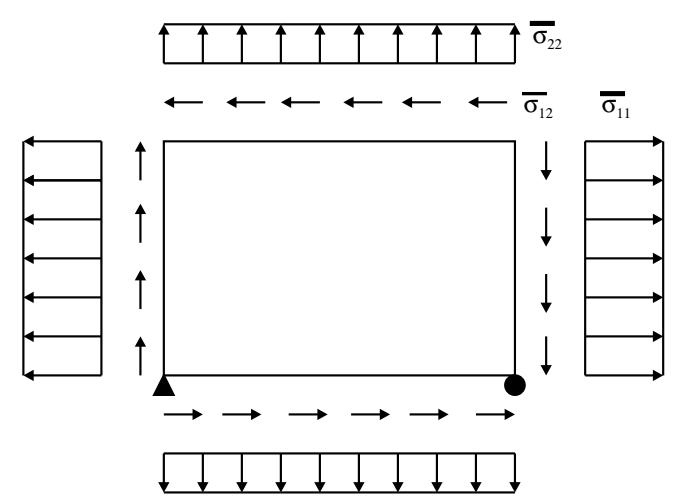

Fig. 3. Geometry and boundary conditions of the considered linear elasticity example in plane stress. 
$e_{22}\left(u^{\tau}\right)=\frac{\bar{\sigma}_{22}}{I_{2}}-\frac{\bar{\sigma}_{11} v}{I_{2}}$

$e_{12}\left(u^{\tau}\right)=-\frac{\bar{\sigma}_{12}(1+v)}{I_{1}}$

where

$I_{1}=\frac{E^{+} E^{-}}{\tau E^{-}+(1-\tau) E^{+}}, \quad I_{2}=\tau E^{+}+(1-\tau) E^{-}$.

So, we have

$$
\begin{aligned}
\chi_{\tau}= & k\left(\rho^{+}-\rho^{-}\right)-\frac{E^{+}-E^{-}}{E^{+} E^{-}}\left(\left(1-v^{2}\right) \bar{\sigma}_{11}^{2}\right. \\
& \left.+E^{+} E^{-}\left(\frac{\bar{\sigma}_{22}-v \bar{\sigma}_{11}}{I_{2}}\right)^{2}+2(1+v) \bar{\sigma}_{12}^{2}\right),
\end{aligned}
$$

which will enable us to determine the analytical solution.

So, let us consider a plate with dimensions 4 times 1 subjected to $\bar{\sigma}_{11}=1, \bar{\sigma}_{22}=0$ and $\bar{\sigma}_{12}=0$. The properties of the base materials are $E^{+}=2, \rho^{+}=2, E^{-}=1, \rho^{-}=1$ and $v=0.25$. For these values, the analytical solution for a rank-1 microstructure without rotation is given by

- if $k \geqslant 0.53125: \bar{\tau}(x)=0$;

- if $k \leqslant 0.484375: \bar{\tau}(x)=1$;

- otherwise: $\bar{\tau}(x)=\sqrt{\frac{2}{32 k-15}}-1$.

Table 2 illustrates the numerical results for three values of $k$, each of them tested for the two types of microstructure, with and without rotation. These are represented just by one element if the design variables take the same value in all elements, where we also indicate the respective values. If the numerical solution is not constant in the domain, we just represent its righthand side.

In all examples we used a mesh with 20 by 20 elements. The exception is example ela-2-10, where we have a mesh of 20 by 40 elements.

The values of $k$ where chosen taking into consideration the qualitative different parts of the presented analytical solution, with particular care to the transition points.

Again, we identify each tested case by a sequence formed of three parts: the first, "ela", identifies the problem type; the second indicates the order in the sequence of the values of $k$; the last one identifies the microstructure type: suffix 10 if rank-1 without rotation $\left(\tau^{(0)}=0.5\right)$, suffix 11 if rank-1 with rotation $\left(\tau^{(0)}=0.5\right.$, $\left.\theta^{(0)}=0\right)$, suffix 20 if rank-2 without rotation $\left(\tau^{(0)}=0.5\right.$, $\left.\mu^{(0)}=0.5\right)$, suffix 21 if rank-2 with rotation $\left(\tau^{(0)}=\right.$ $0.5, \mu^{(0)}=0.5, \theta^{(0)}=0$ ).

For each example, we also indicate the number of iterations the process took to reach convergence (nit) and the value the objective function attained $(j)$.

The obtained numerical solutions for cases 11, 20 and 21 were the expected ones: the solutions are homogeneous and the fibers aligned with the load direction. Two distinct situations occurred regarding case 10: ela-1-10 and ela-3-10 reproduced solutions according with the analytical one. In these two cases the values of $k$ were sufficiently far from the transition points which define

Table 2

Numerical solutions for $k=0.54$ (first line), $k=0.5$ (second line) and $k=0.4$ (third line)

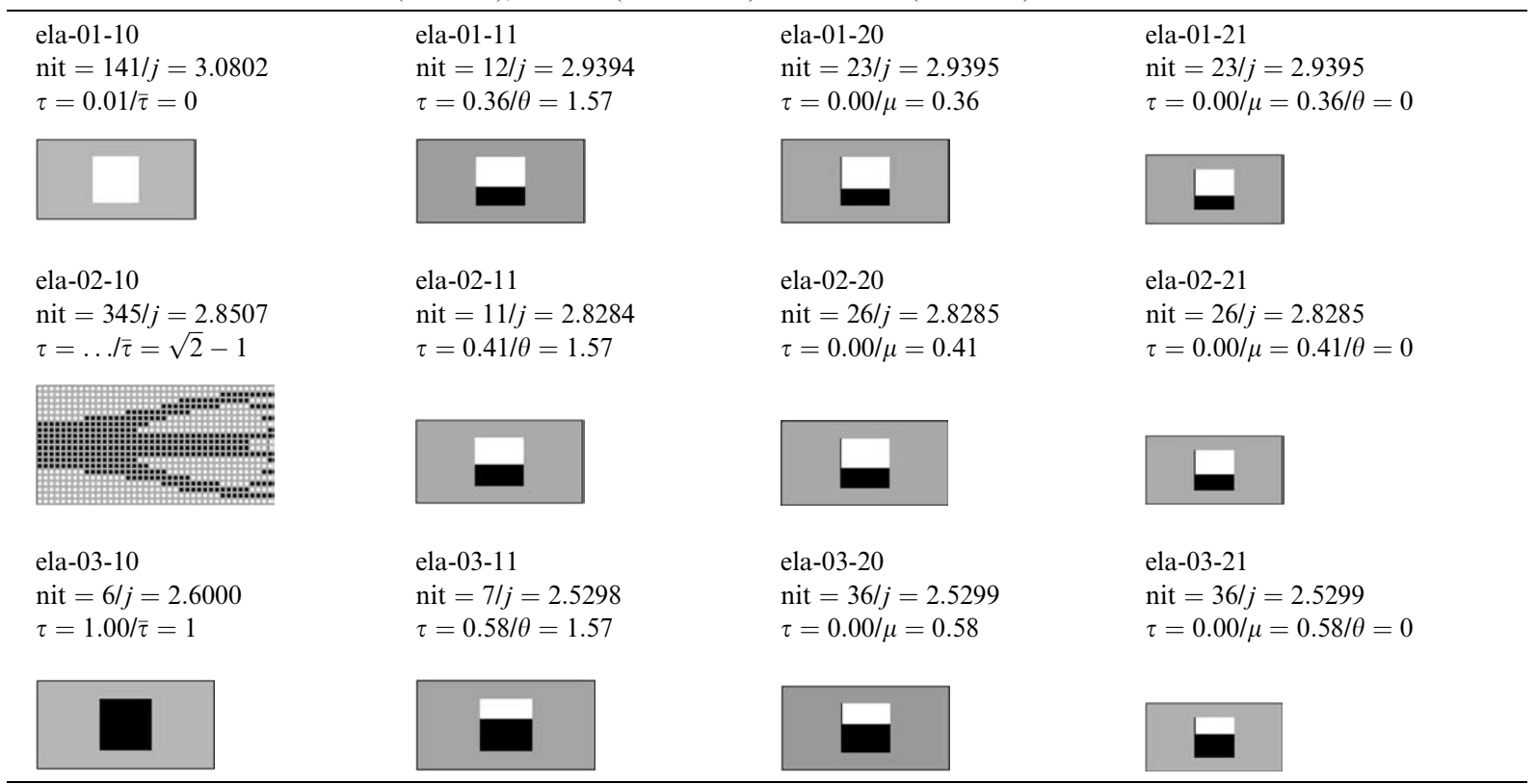


the three branches of the analytical solution. On the other hand, example ela-2-10 produced a non-homogeneous solution. We should note that if we had considered $\tau^{(0)}=\bar{\tau}(=\sqrt{2}-1)$, the iterative process would have stopped after the first iteration. In this case we would have obtained $j=2.9893$, which confirms the obtained result as a better one.

The reason which we identified as the cause to produce better than expected results is the rounding of errors due to float point arithmetic. Indeed, since we are using nine-node quadrilateral elements, for these particular geometry and load conditions and the fact that $\tau^{(0)}$ is a constant vector, the approximation vectors for $e_{11}^{(0)}, e_{22}^{(0)}$ and $e_{12}^{(0)}$ should also be constant in all the domain and equal to the exact values given by (29)-(31), respectively.
This implies that the new approximation $\tau^{(1)}$ should also have been a constant vector. So, the algorithm would produce new approximations till convergence, all of them constant vectors. As this is not what happened, we observe that the algorithm has the ability to take advantage of the rounding errors in such a way that the final solution is a better one, although not homogeneous.

\subsubsection{Bone remodelling}

The example presented in this last section is the application of the presented algorithm to a two-dimensional finite element model of the proximal femur.

The ability of the skeleton to adapt to its functional demands was formally proposed more than a century ago, as outlined in Julius Wolff's 1892 treatise, which hypoth-

Table 3

Bone remodelling example-numerical solution

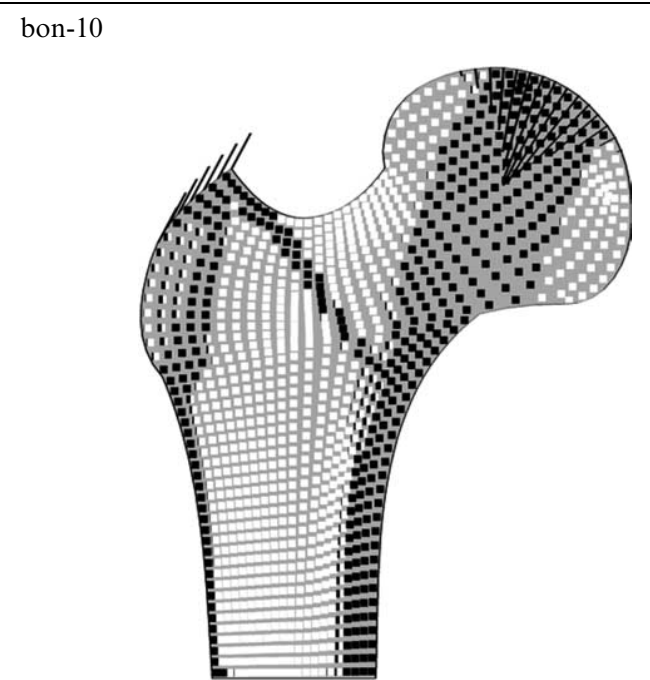

bon-11

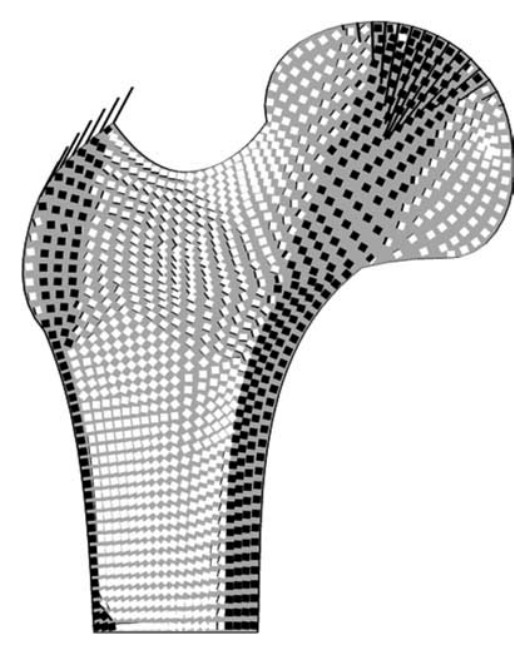

bon-20

bon-21
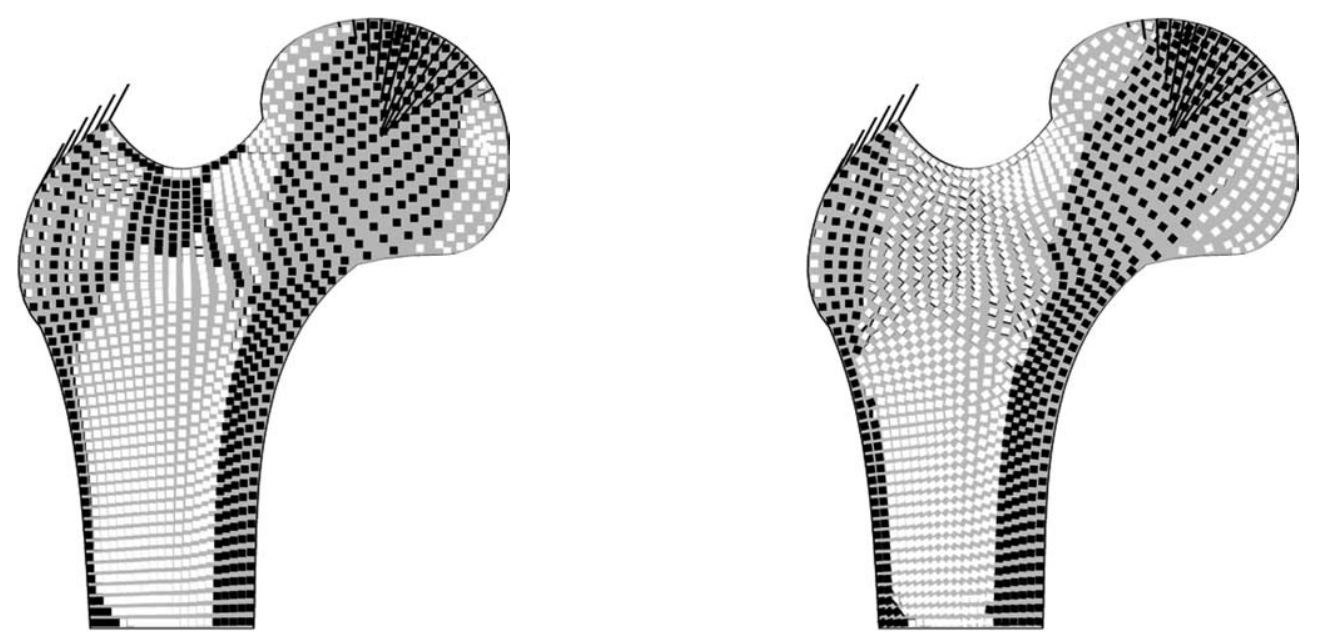
esized that the form and interior constitution of bone is a product of alterations in its internal architecture according to a kind of intrinsic mathematical rules. The main point of Wolff's Law-bone remodelling tends towards optimizing (minimal mass/maximal stiffness) structural criteria of the skeleton, has gained wide acceptance.

The model presented gives: first, the objective function (16) expresses the conflict between the minimization of stress and minimization of mass identified in Wollf's Law; second, the use of laminates allows for the exact calculation of the homogenized coefficients and to reproduce a kind of oriented trabeculae where rotation of the laminate is enabled.

Table 3 illustrates the numerical results for a finite element mesh with 1144 isoparametric $C^{0}$ quadrilateral eight-node elements and a side-plate [14] with 567 nondesign elements, in plane strain.

In this example we identify each tested case by a sequence formed of two parts: the first, "bon", identifies the problem type; the second identifies the microstructure type and if rotation is allowed or not.

The considered properties of the based materials were $E^{+}=14,500 \mathrm{~N} / \mathrm{mm}^{2}, \rho^{+}=174 \mathrm{e}-2 \mathrm{~g} / \mathrm{mm}^{3}, E^{-}=500 \mathrm{~N} /$ $\mathrm{mm}^{2}, \rho^{-}=30 \mathrm{e}-2 \mathrm{~g} / \mathrm{mm}^{3}$ and $v=0.2$. The loads, measured in $\mathrm{N} / \mathrm{mm}^{2}$ and which represent a day-by-day situation, are represented in the figures by black lines proportionally to their values. The obtained results are qualitatively in accordance with the expected ones. However, we should note that when rotation is enabled, the laminate orientates with the principal strains. This is due to the fact the material is anisotropic, where there is not an agreement between principal stresses and principal strains.

\section{Acknowledgements}

The authors would like to thank the anonymous referee for valuable comments and suggestions that improved the content of the paper.

\section{References}

[1] Allaire G. Shape optimization by the homogenization method. Springer; 2002.

[2] Bagge M. A model of bone adaptation as an optimization process. J Biomech 2000;33(11):1349-57.

[3] Bendsøe MP. Optimal shape design as a material distribution problem. Struct Optim 1989;1:139-202.

[4] Bendsøe MP. Optimization of sructural topology, shape and material. Springer; 1995.

[5] Bendsøe MP, Kikuchi N. Generating optimal topologies in structural design using a homogenization method. Comput Methods Appl Mech Eng 1988;71:192-224.

[6] Bensoussan A, Lions JL, Papanicolau G. Asymptotic analysis for periodic structures. In: Studies in Mathematics and its Applications. North-Holland; 1978.

[7] Díaz A, Sigmund O. Shape optimization of structures for multiple loading conditions using a homogenization method. Struct Optim 1992;4:17-22.

[8] Díaz A, Sigmund O. Checkerboard patterns in layout optimization. Struct Optim 1995;10:40-5.

[9] Fernandes P, Rodrigues HC, Jacobs CR. A model of bone adaptation using a global optimization criterion based on the trajectorial theory of Wolff. Comput Methods Biomech Biomed Eng 1998;2:125-38.

[10] Murat F, Tartar L. H-convergence, Séminaire d'analyse fonctionnelle et numérique de l'Université d'Alger 197778, mimeographed, 1997.

[11] Pedersen P. Optimal orientation of orthotropic materials. Struct Optim 1989;1:101-6.

[12] Pedersen P, Bendsøe MP. Synthesis in bio solid mechanics. Kluwer Academic Publishers; 1999.

[13] Rubin CT. Physical and environmental influences on bone formation. In: Brighton CT, Friedlaender G, Lane JM, editors. Bone formation and repair. Rosemont, IL: AAOS; 1994.

[14] Verdonschot N, Huiskes R. Fem analysis of hip prostheses: validity of the 2-d side-plate model and the effects of torsion. In: 7th Meeting of the European Society of Biomechanics, Aarhus, Dinamarca, 1990.

[15] Wolff J. The law of bone remodelling (Das Gesetz der Transformation der Knochen, Hirschwald, 1892) [Maquet P, Furlong R, Trans.]. Springer, Berlin, 1986. 